\title{
Robust Period Estimation of Automated Cutting Systems by Improved Autocorrelation \& Linear Regression Techniques
}

\author{
Anthony McAtear \\ IDLab, Dept. Electronics \& \\ Information Systems \\ Ghent University - imec, Belgium \\ Anthony.McAtear@UGent.be
}

\author{
Ruben Gielen \\ Production Services \\ Aperam Stainless Steel, Belgium \\ Ruben.Gielen@aperam.com
}

\author{
Nilesh Madhu \\ IDLab, Dept. Electronics \& \\ Information Systems \\ Ghent University - imec, Belgium \\ Nilesh.Madhu@UGent.be
}

\begin{abstract}
Condition monitoring is an important asset in the industry to improve the safety and efficiency of the production chain. However, in heavy machinery - such as edge trimmers in steel mills - it is often impractical and unsafe to install intrusive sensors to get the data needed for condition monitoring. Nonintrusive monitoring techniques based, e.g., on acoustic data captured by microphones placed in the vicinity of the assembly being monitored are attractive options. Our application deals with the acoustic monitoring of rotational blades cutting steel strips at high speeds. Knowing the correct period of the cutting process is important for quality evaluation purposes. We propose two novel robust methods to estimate the periodicity based on the audio captured by a microphone near the blades. One is an improved autocorrelation function and the other is based on linear regression, both using incorporating an novel test for the correctness of the estimated period. We compare our methods against the standard autocorrelation-based periodicity measurement techniques on real data recordings. The proposed method estimates the correct period about $87 \%$ of the time, compared to an accuracy of only $51 \%$ using standard periodicity measurement approaches.
\end{abstract}

Index Terms-Period estimation, linear regression, autocorrelation, condition monitoring, non-intrusive system parameter estimation

\section{INTRODUCTION}

Condition monitoring is an important asset in the industry to improve the safety and production chain efficiency. We are working on a particular aspect of this in the steel industry. Here, in the manufacture of the steel plates and coils, an edge trimming line is used in the final stages for cutting the steel roll to its final size. The waste portions are chopped into strips of uniform length for recycling. This chopping of the waste is done by a rotational blade assembly. In Fig. 1a an edge trimming line is schematically depicted and in Fig. $1 \mathrm{~b}$ a graphic of the chopper blades is shown.

The cutting of steel wears the blades out very quickly, and being able to predict any kind of failures of the cutting equipment is a big benefit. However, for such industrial machinery it is often impractical to integrate sensors into the core of the machine, due to the infrastructure that is already in place for safety and/or operational reasons. In these situations, non-intrusive sensors such as microphones or cameras need to be used. Here we deal with the monitoring of

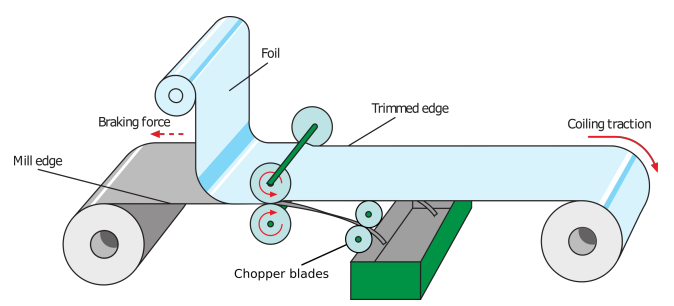

(a) A simplified schematic of the edge trimming line

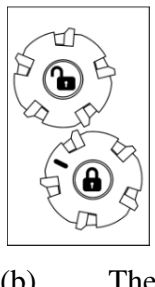

(b) The chopper blades
Fig. 1: The edge trimming line is shown in (a). In (b), a side view of the chopper blades are shown.

the chopper blades of a process line that trims different steel types with thicknesses ranging from about 3 to 8 millimeter. For this we use a microphone mounted half a meter from the chopper blades. To facilitate temporal cut localization for further processing, the period between two cuts is estimated. However, the cutting is not guaranteed to be perfectly periodic due to play of the waste.

Period estimation has been tackled in literature using several different techniques and for different use cases. Periodicity mining algorithms, such as in [1], [2], attempt to extract the period of periodic patterns from a simplified sequence obtained by mapping the time series data. This mapping, often referred to as symbolization, compresses the signal in time domain, inevitably lowering the sample accuracy that can be achieved. The autocorrelation (AC) function is also extensively used for period estimation. For example, it can be used to extract the pitch period of speech signals by finding the highest peak in the AC. In [3], a weighted AC function is used. The weighting is done using the average magnitude difference function (AMDF) which has a notch where the lag is equal to the period. However, depending on the state of the blades, both $\mathrm{AC}$ and $\mathrm{AMDF}$ would either find the interval between two cuts or that of a full rotation, and is thus not reliable. In [4], Nishiguchi and Kobayashi propose an improved algorithm making use of the $\mathrm{AC}$ function for estimating the interval between pulses of a pulse train. However, this is not applicable to our recordings because there is no established way of converting the audio into a pulse train. 
In this work we propose two novel methods to estimate the interval between two cuts, the first based on the AC function and the other based on linear regression (LR). To improve the robustness of the approaches and to further reduce errors in the period estimate, several candidate period values are computed and a so-called variance test test is applied to determine the best estimate.

The paper is organized as follows: Section II describes the signal model and a standard method for period estimation using the AC function. In section III we propose an improved $\mathrm{AC}$ function, together with a method to test the correctness of an estimate of the period. Based on this, we next propose a new method using linear regression in section IV. Section V demonstrates how both proposed methods can be combined. The results are discussed in section VI and finally, section VII presents the conclusions and future work.

\section{Period ESTIMATION}

Period estimation attempts to extract the period $T$ from a periodic signal. The estimated period $\widehat{T}$ is given by $\widehat{T}=T-\epsilon$, with $\epsilon$ the error between the estimate and the correct period.

\section{A. Signal model}

The cutting of steel in our situation produces a periodic signal when the speed is constant. During a cut, the captured audio signal has high energy and thus also high magnitude peaks. This can be seen in Fig. 2, where the cut regions are marked in grey in the time domain signal on top. In the spectrogram below, the high energy regions can be seen as bright vertical strips. The audio signal $s(n)$ (captured at a sample rate of $f_{s}=48 \mathrm{kHz}$ ) thus has a period given by the interval between two cuts, denoted as $T$. Each periodic segment is referred to as an epoch. Depending on the condition of the blades, the individual epochs may be slightly irregular with respect to each other, but the pattern will still repeat over the full rotation of the blade assembly. Further, one full rotation of the blade assembly will cut the steel $E$ times, where $E$ indicates the number of blades in the assembly. Thus a rotation consists of $E$ epochs, referred to as a sweep. In some cases, the control algorithm changes the speed of the choppers slightly, however this change cannot be detected from the measured speed data itself. This results in the signal not being perfectly periodic but near-periodic.

\section{B. Standard autocorrelation}

Autocorrelation is widely used to extract the period of a periodic signal. The discrete AC function is given by

$$
R_{s s}(\tau)=\sum_{n} s(n) s(n+\tau)
$$

with $\tau$ denoting the lag between the original and the shifted version of the signal. The estimate of the period $\widehat{T}$ is given by the lag $\tau$ that corresponds with the highest magnitude peak of the $\mathrm{AC}$ of a periodic signal, i.e. $\tau \simeq T$.

$$
\widehat{T}=\underset{\tau}{\arg \max } R_{s s}(\tau)
$$

The $R_{s s}(\tau)$ depends on the magnitude of the signal so it can be biased by the high-energy parts of the signal that do not
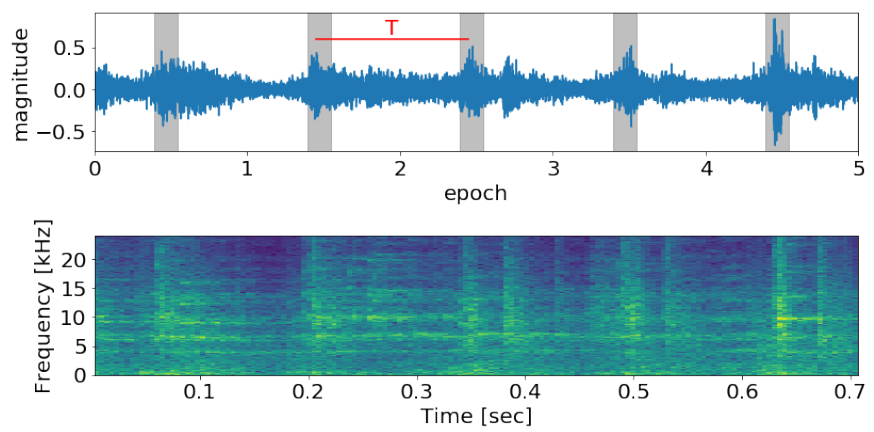

Fig. 2: A periodic segment with a length of 5 epochs, where one epoch is the same length as the period $T$. The top shows the time domain signal where the cutting of the steel is marked in grey. The bottom graph shows the spectrogram of the signal. The signal is very noisy and this noise is extremely nonstationary due to constantly falling scrap metal and other noise sources. Further, note that the time axis of the top plot is in number of epochs, while it is in seconds for the spectrogram. This gives an idea of the time scales involved.

come from the cutting but are caused by the background noise. This makes the period estimation difficult. Another drawback of the AC is that the lag which corresponds to the biggest peak is not necessarily the period of the signal, but rather an integer multiple of it, especially when the signal is quasi-periodic as is the case here. This phenomenon is known as "octave error" [5]. Also, for small values of the lag $\tau$, the correlation will be significantly higher than the peak where $\tau \simeq T$, which means that finding small periods becomes less reliable. To prevent this, a priori knowledge of the maximum chopper speed, which corresponds to the minimal period $T_{\min }=3000$, is used to limit the search range to $\tau \geq T_{\min }$. The standard AC is contrasted with our proposed method in the results section.

\section{IMPROVED AUTOCORRELATION FUNCTION}

One of the shortcomings of the AC function is that it is possible that the peak corresponds with an integer multiple of the period rather than the period itself. Here we propose an improved AC (IAC) method to determine the best period estimate from a set of $K$ candidate periods. These candidates are given by $\widehat{T}_{k}=\widehat{T} / k$, for $k=1, \ldots, K$, where $\widehat{T}$ is the initial estimate obtained from (2). However, the search range is further constrained to $4 \cdot T_{\text {min }} \leq \tau \leq K \cdot T_{\text {min }}$.

\section{A. Matrix representation of a periodic signal}

The first step of this approach is a transformation of the one-dimensional periodic signal $s(n)$ into a two-dimensional matrix $\boldsymbol{S}(\widehat{T})$.The transformed matrix $\boldsymbol{S}(\widehat{T})$ has dimensions $(N \times L)$, with $N=\lfloor\widehat{T}\rfloor$ the number of samples per epoch, and $L=N_{s} /\lfloor\widehat{T}\rfloor$ the number of epochs. $\lfloor\bullet\rfloor$ denotes the floor function. This means that the $l^{\text {th }}$ column of $\boldsymbol{S}(\widehat{T})$ corresponds to the $l^{\text {th }}$ epoch of $s(n)$. If the transformation is performed using the correct period $T$, i.e. $\boldsymbol{S}(T)$, then all epochs will be perfectly aligned. For this we exploit the fact that the signal segment should ideally (in noiseless conditions) exhibit a region of high intensity in the vicinity of a cut and low energy elsewhere. Thus, if the signal is "epoched" at the 
correct period, all the high-intensity segments would line up across the different epochs. When $T$ has a nonzero fractional part $\{T\}$, one sample is removed every $\{T\}^{-1}$ epochs to correct for this. Also, to ensure that the high energy regions are primarily in the middle of the epoch, the signal is shifted by the circular mean of the maxima positions of each epoch. Such a corrected matrix $|\boldsymbol{S}(T)|$ is shown in Fig. 3 (a). The dark line

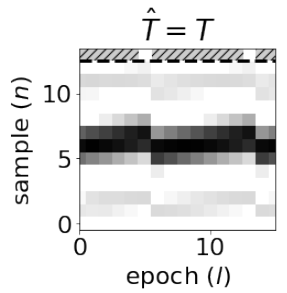

(a)

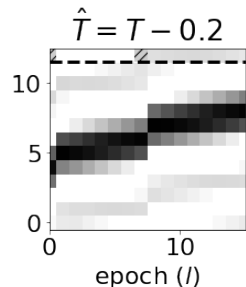

(b)

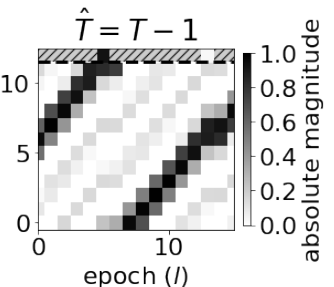

(c)
Fig. 3: The absolute matrix representation $|\boldsymbol{S}(\widehat{T})|$ of a periodic signal with period $T=13+1 / 7$. The hatched region above the dashed line is where no samples are removed, the other pixels above this line are the samples that are removed to correct for the fractional period. In (a), $\widehat{T}=T$, in (b) there is an error $\epsilon=0.2$ and in (c) the error is equal to 1 .

indicates regions of high intensity and is horizontal because the transformation was done with the correct period $T$. In (b), the line has a slope due to the error $\epsilon=0.2$ that is introduced. This means that for every epoch 0.2 samples are moved to the next epoch. After 10 epochs, the high magnitude region will have moved up by 2 full samples. The error is increased to $\epsilon=1$ in (c), causing the line to wraparound from top to bottom.

\section{B. Variance test}

The alignment of the high intensity regions around the cut can be used to check the correctness of the period. When the high intensity line is horizontal, the maxima positions of each epoch will be roughly equal. On the other hand, when there is a slope (indicating inaccurate $\widehat{T}$ ), the maxima positions will be spread out. This means we can select the best period estimate based on the variance of the maxima positions of the transformation $\boldsymbol{S}(\widehat{T})$. The maxima positions of a transformation by $\widehat{T}$ is given by

$$
y_{k}(l)=\underset{n}{\arg \max }\left|S_{n, l}\left(\widehat{T}_{k}\right)\right| \forall l \in 1, \ldots, L,
$$

where $S_{n, l}(\widehat{T})$ indicates a single sample of the matrix $\boldsymbol{S}(\widehat{T})$ at the $n^{\text {th }}$ sample of the $l^{\text {th }}$ epoch. Testing the period estimates $\widehat{T}_{k}$ is thus possible by calculating the normalized variance

$$
\nu_{k}=\frac{\operatorname{var}\left(\boldsymbol{y}_{k}\right)}{\widehat{T}_{k}^{2}} \text {. }
$$

The variance is normalized to ensure it is comparable for different periods. The best estimate $\widehat{T}$ is thus the one for which $\nu_{k}$ is smallest: $\widehat{T}=\widehat{T}_{k^{\prime}}, k^{\prime}=\arg \min _{k} \nu_{k}$.

In figure 4 , the normalized variance is plotted in function of the estimated period $\widehat{T}$. The normalized variance is significantly lower when the period is equal to the correct period $T$. Ideally, the normalized variance should be high everywhere other than the correct period. However, as $\widehat{T}$ increases, the normalized variance decreases. This is likely due to the fact that the normalized variance is calculated with fewer maxima positions for larger values of $\widehat{T}$.

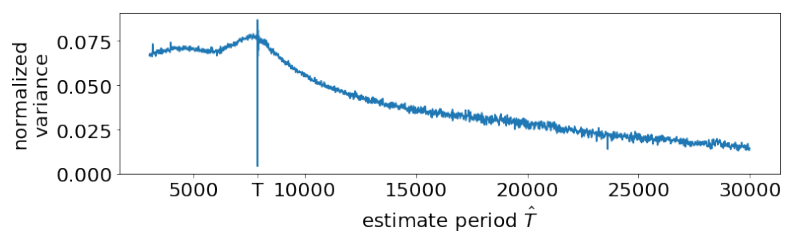

Fig. 4: A plot of the normalized variance in function of the period. $T=7870$ is the correct period, where the variance is at its lowest. However, as the estimate period $\widehat{T}$ increases, the normalized variance decreases, which is undesirable.

\section{LINEAR REGRESSION APPROACH}

In the previous section we see that the matrix representation can be used to determine whether the period is correct or not, based on the slope of the line. If the period is not correct the line has a slope which is equal to the error $\epsilon$. This means that when we can estimate the slope, we can find the correct period by simply calculating $T=\widehat{T}+\epsilon$.

Therefore we introduce a new method that does not rely on the AC but on linear regression (LR). Another advantage of this LR approach is that it only relies on the position of the high intensity regions and not on the exact magnitude. Thereby, this approach is less biased by signal segments which are heavily corrupted by noise (unlike the AC method). However, the initial period estimate needs to be within a certain range of the correct period for this method to converge. Here too we make use of a priori information to limit the range of possible period candidates between $T_{\min }$ and $T_{\max }$. To cover any period $T$ within this range, we make use of $K$ initial period estimates $\widehat{T}_{k}^{(0)}$. Finally, the variance test proposed before can be used here as well to select which period estimate is the best.

\section{A. Transformation in terms of sweeps}

Since epochs can be irregular with respect to each other depending on the state of the blades, it is of interest to work with sweeps rather than epochs. This results in the transformation $S(E \widehat{T})$ of size $(\lfloor E \widehat{T}\rfloor \times L)$ with $E$ being the number of epochs in one sweep. To simplify the mathematical expressions, $S(E \widehat{T})$ is further partitioned into $E$ parts forming a tensor of size $(N \times L \times E)$ denoted as $S^{\prime}(\widehat{T})$. The element $S_{n, l, e}^{\prime}(\widehat{T})$ of tensor $S^{\prime}(\widehat{T})$ refers to the sample at position $n$ of the $e^{t h}$ epoch of sweep $l$.

Each partition has a line similar to before, but unlike the previous case, the line is formed by the cuts of a single blade instead of all 5 blades. However, since the signal is transformed by $E \widehat{T}=E(T-\epsilon)$, the error $\epsilon$ is multiplied by $E$ as well, resulting in a slope that is $E$ times bigger. The period correction thus becomes

$$
T=\widehat{T}+\frac{m}{E}
$$

with $m$ the slope of the lines. 


\section{B. Wraparound removal}

Linear regression requires the lines to be straight without wraparound, otherwise the fitted line will be nowhere near the correct slope. To do this we find a rough estimate of the slope by looking at the difference of the maxima positions between consecutive sweeps. The maxima positions are now defined as

$$
Y_{l, e}=\underset{n}{\arg \max }\left|S_{n, l, e}^{\prime}(\widehat{T})\right|+(e-1) N,
$$

for all epochs $e=1, \ldots, E$ in all sweeps $l=1, \ldots, L$. The added term $(e-1) N$ is there so that the maxima position is relative to the start of the sweep rather than the start of the epoch. The difference $D_{l, b}$ between each maximum and the closest maximum of the next sweep is then given by

$$
D_{l, e}=Y_{l+1, \beta}-Y_{l, e},
$$

with $\beta=\arg \min _{j}\left|Y_{l, e}-Y_{l+1, j}\right|$ for $j=1, \ldots, E$. The wraparound effect will cause many of the differences $\boldsymbol{D}$ to be the wrong slope, however, the most frequently occurring slope $d$ will most likely be close to the correct slope, which is used to update the estimated period to a better period estimate

$$
\widehat{T}^{(i+1)}=\widehat{T}^{(i)}+\frac{d}{E},
$$

with $\bullet^{(i)}$ denoting the iteration. Repeating this step can improve the period estimate further since there will be less wraparound, and thus the differences $\boldsymbol{D}$ will be less affected by it.

Wraparound can only be removed when the initial error is not too high. This means that the range in which an initial period $\widehat{T}_{k}^{(0)}$ must lie is given by $\left[T-\frac{T \alpha_{\max }}{2}, T+\frac{T \alpha_{\max }}{2}\right]$, with $\alpha_{\max }=\frac{1}{E}$. This range is referred to the convergence region. The bounds of the convergence region are defined due to the fact that when the absolute error is larger than $\frac{T}{E}$, the slope will be more than half the period. However, because we find the difference between the closest consecutive maxima, the slope cannot be larger than half the period.

Given we know the convergence range, a set of initial periods $\widehat{T}_{k}^{(0)}$ can be defined to cover the search range $\left[T_{\min }, T_{\max }\right]$ so that at least one of the initial periods will converge to the actual period $T$. To minimize the number of initial periods, and thus also the computational complexity, we use the following function $\widehat{T}_{k}^{(0)}=T_{\min } \cdot(1+\alpha)^{k-1}$, with $k=1, \ldots, K$ and $0<\alpha<\alpha_{\max }$ a measure of how densely the initial periods are chosen within the given range. Choosing a small value for $\alpha$ increases computation time, however, a too large value of $\alpha$ may risk missing the value in the range of the true period. We have empirically found $\alpha=\frac{1}{2.5 E}$ good for our purpose.

\section{Period fine tuning using linear regression}

Now that there is at least one period estimate where the wraparound is successfully removed, linear regression can be used to find the slope $m$ more accurately to improve this period estimate further. Here we also make use of the transformation based on sweeps $S^{\prime}(\widehat{T})$ and the maxima positions $\boldsymbol{Y}$ given in (6). This gives us $E$ sets of points that lie in a line over all sweeps. Each set corresponds to one epoch. The slope of these lines will be the same since the speed of the choppers is constant, so we want to find the shared slope $m$. The yintercept however, will be different for each set of points, and is thus a row vector of length $E$ denoted by $c^{T}$.

The slope $m$ can be derived from the least squares equation

$$
\mathcal{J}=\sum_{e=1}^{E} \sum_{l=1}^{L}\left(Y_{l, b}^{\prime}-Y_{l, b}\right)^{2},
$$

where we try to minimize the squared difference between the line $\boldsymbol{Y}^{\prime}=m \boldsymbol{X}+\boldsymbol{c}^{T}$ and the maxima positions $\boldsymbol{Y}$. By the standard formulae for linear regression, the slope $m$ for which $\mathcal{J}$ is minimum is given by

$$
m=\frac{\sum_{b} \operatorname{cov}(\boldsymbol{X}, \boldsymbol{Y})}{\sum_{b} \operatorname{var}(\boldsymbol{X})},
$$

with $\boldsymbol{X}$ and $\boldsymbol{Y}$ the $\mathrm{x}$ and $\mathrm{y}$ coordinates of the maxima positions. The estimated period is corrected by the slope $m$ as defined in (5).

The wraparound removal and the period fine tuning steps are done for all $K$ initial period estimates. Subsequently, we can use the variance test proposed in III-B, to select the best period estimate.

\section{COMBINED PERIOD ESTIMATION METHOD}

We now have two period estimation algorithms, one uses an improved autocorrelation method and the other makes use of linear regression. Both these methods utilize the variance test to determine the best period estimate from a set of estimates. The variance test is completely separate from the method used to estimate the periods, and thus can also be used to determine the best period estimate from two or more estimates obtained using different methods. This hybrid period estimation method is just that, where we select the best estimate of either the IAC or LR approach as the one with the lowest variance.

\section{RESUlTS}

In this section we discuss and compare the results from our novel proposed methods based on improved AC and LR, as well as the standard AC method. The data used for evaluation are 102 periodic regions taken from 80 real recordings. A second order, zero phase high-pass filter with a cutoff of $100 \mathrm{~Hz}$ is applied on the raw signal, which is then squared and followed by a recursive smoothing filter with smoothing parameter $\alpha=0.98$. The number of blades in the assembly $E$ is equal to 5 . The periodic regions are of varying length, ranging from around 50 epochs to 2000 epochs, with most of the regions being around 300 epochs long. These methods are evaluated using the ground truth data obtained by converting the speed of the chopper into the period in samples. Based on the matrix representation, we can determine the accuracy of the different period estimation methods, but also that of the ground truth (GT). We find that the GT slightly varies around the real period with about 10 samples. For this reason, the interpretation of these results should be done with caution.

In Fig. 5, the results of AC, IAC and LR are plotted against the GT period. We see that the improved AC and the LR method have a significantly larger number of correctly estimated periods (i.e. points that lie on the $\mathrm{x} 1$ line). The 
standard $\mathrm{AC}$ has a substantial amount of estimations on the x 5 line, i.e. estimating the sweep, but also other multiples of the GT period. This is not the case for IAC and LR, but do suffer from estimations that are half of what they should be.

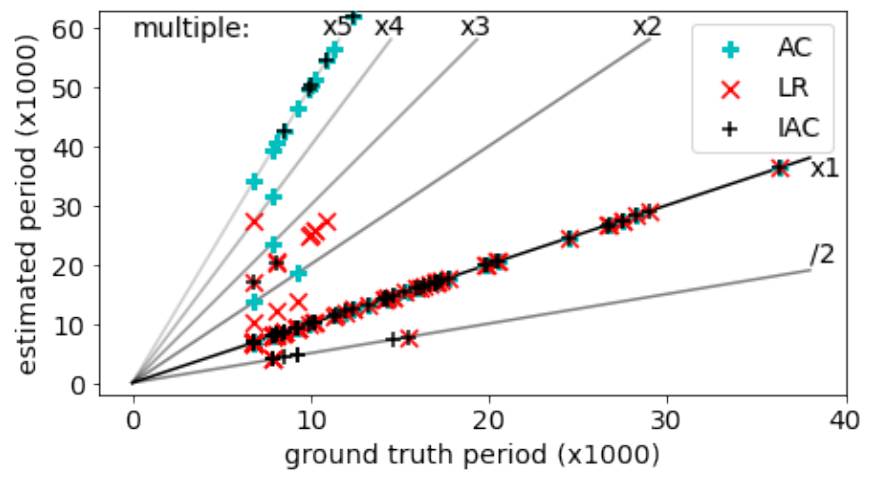

Fig. 5: A comparison between the $\mathrm{AC}$ and the two novel proposed methods IAC \& LR, plotted against the ground truth (GT) period. The lines mark different multiples of the GT.

The detection rates for different multiples of the ground truth are summarized in table I. It shows the number of estimations that are within 100 samples of a multiple of the GT period. Here we clearly see the improvement that IAC gives over AC. Nearly all estimates of AC that are multiples of the GT period are correctly estimated by IAC. The LR method also does not seem to suffer from estimating multiples of the GT period and has more correct estimates than IAC. We can also see that the combined method has an even higher accuracy rate than either IAC or LR of around $87 \%$.

\begin{tabular}{r|ccccccc} 
& $/ 2$ & $\mathrm{x} 1$ & $\mathrm{x} 2$ & $\mathrm{x} 3$ & $\mathrm{x} 4$ & $\mathrm{x} 5$ & other \\
\hline AC & 0 & 52 & 7 & 1 & 1 & 34 & 7 \\
IAC & 8 & 79 & 0 & 0 & 0 & 5 & 10 \\
LR & 4 & 86 & 0 & 0 & 1 & 0 & 11 \\
Combined & 7 & 89 & 0 & 0 & 0 & 3 & 3
\end{tabular}

TABLE I: The detection rates of the different algorithms. It shows the number of estimations that are within 100 samples of a multiple of the ground truth (GT) period.

It is further important to note that from the correctly estimated periods of the standard AC method, 4 are incorrectly estimated by both the proposed methods, and thus also by the combined method. This means that in some cases the variance test does not always perform optimally.

The RMSE of the correctly estimated periods is 19.2 samples for AC, 17.8 for IAC, 19.7 for LR and 18.4 for the combined method. To get a better idea of the accuracy, we used the matrix representations to manually check the error. We find that the absolute error is less than 6 samples for the combined method when the signal has 100 or more epochs. For signals that only have around 40 epochs, the error goes up to 70 samples. Two of these matrix visualizations are shown in Fig. 6. (a) shows a short periodic region, while (b) shows a near-periodic but longer region. The upward bend indicates a speed reduction but this only accounts for a change in period of 3 samples $(=62.5 \mathrm{~ms})$. Here the estimated period is the average period of the signal.
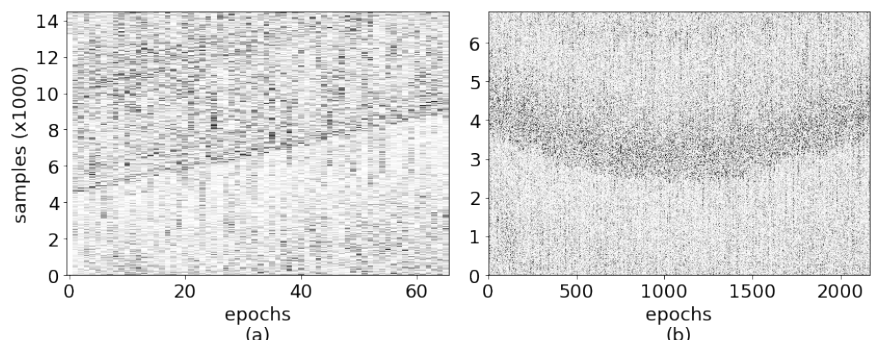

(a)

(b)

Fig. 6: The matrix representation $S(\widehat{T})$ of two recorded signals. In (a), the signal is perfectly periodic but short. It is harder to estimate the period of short segments. Here the error is about 70 samples. The signal in (b) is near-periodic, with a deviation of about 3 samples.

\section{CONCLUSIONS}

Period estimation in low SNR conditions is not a trivial problem. We exploited the high intensity region around a cut to align the different epochs of the recorded signal in a 2$\mathrm{D}$ representation. We further proposed a novel method for period estimation based on linear regression as well as an improved AC method. Also, a test to assess the correctness of the period is proposed using the variance statistic. Results of real recordings demonstrate that the combined method of the two newly proposed algorithms can estimate the period very accurately $87 \%$ of the time, compared to about $51 \%$ using the standard AC method. These results can possibly be improved further by applying stationary noise suppression algorithms to enhance the signal, such as [6], [7]. Also outlier removal algorithms can be used to improve the accuracy and detection rates. This will be studied in future work.

\section{ACKNOWLEDGMENTS}

This research has been carried out in the framework of the TOBIAS project which has received funding from the Flanders Innovation \& Entrepreneurship (VLAIO), Ghent University and imec.

\section{REFERENCES}

[1] M. G. Elfeky, W. G. Aref, and A. K. Elmagarmid. Periodicity detection in time series databases. IEEE Transactions on Knowledge and Data Engineering, 17(7):875-887, July 2005.

[2] F. Rasheed, M. Alshalalfa, and R. Alhajj. Efficient periodicity mining in time series databases using suffix trees. IEEE Transactions on Knowledge and Data Engineering, 23(1):79-94, Jan 2011.

[3] S. Kumar. Performance measurement of a novel pitch detection scheme based on weighted autocorrelation for speech signals. International Journal of Speech Technology, 22(4):885-892, 2019.

[4] K. Nishiguchi and M. Kobayashi. Improved algorithm for estimating pulse repetition intervals. IEEE Transactions on Aerospace and Electronic Systems, 36(2):407-421, April 2000.

[5] Q. Lin and Y. Shao. A novel normalization method for autocorrelation function for pitch detection and for speech activity detection. In Interspeech, pages 2097-2101, 2018.

[6] Y. Ephraim and D. Malah. Speech enhancement using a minimum mean-square error log-spectral amplitude estimator. IEEE transactions on acoustics, speech, and signal processing, 33(2):443-445, 1985.

[7] J. S. Erkelens, R. C. Hendriks, R. Heusdens, and J. Jensen. Minimum mean-square error estimation of discrete fourier coefficients with generalized gamma priors. IEEE Transactions on Audio, Speech, and Language Processing, 15(6):1741-1752, 2007. 